\title{
GENETIC ENGINEERING
}

\section{CRISPR improves prospects for transgenic rats}

Bäck, S. et al. Neuron 102, 105-119.e8 (2019)

Depending on whom you ask, rats are more complex rodents than mice. They can be trained to perform more challenging cognitive tasks and have a richer repertoire of behavior and social interactions. For a time they were king of the lab, particularly for behavior and neuroscience-related research. Then came genome engineering, and mice took the throne.

In the early days, results could be random-researchers would often have to make and screen many animals to find those that carried a desired genetic mutation. Mice had some technical advantages over rats, but also a practical one, says Brandon Harvey of the National Institutes of Health: cheaper per diems. Compounding things, the mouse genome was completed two years before that of the rat. Rats have been playing catch-up ever since, he says.

But the rodent playing field may be leveling. In work published in Neuron, Harvey and colleagues at the NIH and University of Texas Southwestern
Medical Center use CRISPR-Cas9 technology to engineer rats with targeted edits to mature neurons.

Overall, CRISPR has made the genome editing process much more efficient, Harvey says. In the paper, they edit both embryonic and spermatogonial stem cells-the latter can be injected into ablated males to breed transgenic progeny directly. The researchers also built several rat lines in which genome editing can occur within adult cells by combining the Cre system, which enables conditional editing, with CRISPR-Cas9 constructs that guide where editing should occur. They demonstrate applications to dopaminergic neurons but theoretically any cell can be targeted, Harvey says.

There is still room to improve the process in rats; the next phase will include testing whether CRISPR modifications such as CRISPRa and CRISPRi can modulate gene expression more precisely, Harvey says. Meanwhile, the new rat lines will be used to study gene function in the adult brain in his lab, which studies the mechanisms of dopaminergic neuron dysfunction in models of substance abuse.

Is it time for the return of the rat? "I think we'll continue to see the emergence of more rat models," Harvey says. "It's just a matter of how accessible they'll be to investigators." On that point, he and his colleagues demonstrate different approaches labs can take: transgenic animals can be bred and maintained in a colony, or the tools can be delivered via viral vectors as needed. While technology may be less of a limitation, funding still can be. It's gotten easier to make transgenic rats with fewer animals, but a rat remains more expensive than a mouse. Other labs Harvey has talked to are excited about the prospect of returning to rats, but rat research needs resources.

\section{Ellen P. Neff}

Published online: 21 May 2019

https://doi.org/10.1038/s41684-019-0316-8

\section{What Control Diet are you using?}
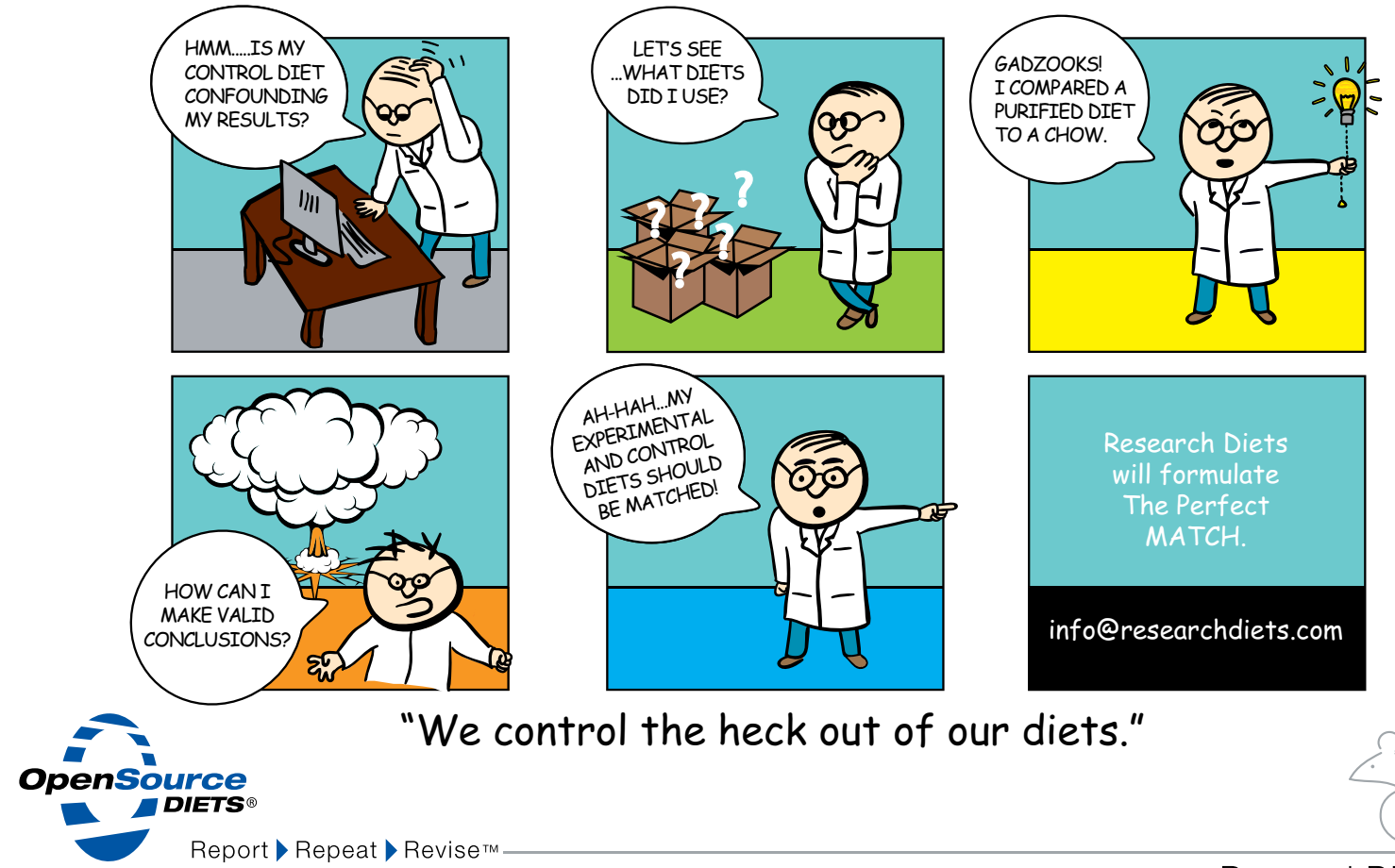